\title{
3c 220.3: A Radio Galaxy Lensing a Submillimeter Galaxy
}

\section{Citation}

Haas, Martin, Christian Leipski, Peter Barthel, Belinda J. Wilkes, Simona Vegetti, R. Shane Bussmann, S. P. Willner, et al. 2014. "3c 220.3: A Radio Galaxy Lensing a Submillimeter Galaxy." The Astrophysical Journal 790 (1) (July 2): 46. doi:10.1088/0004-637x/790/1/46.

\section{Published Version}

doi:10.1088/0004-637X/790/1/46

\section{Permanent link}

http://nrs.harvard.edu/urn-3:HUL.InstRepos:30212124

\section{Terms of Use}

This article was downloaded from Harvard University's DASH repository, and is made available under the terms and conditions applicable to Other Posted Material, as set forth at http:// nrs.harvard.edu/urn-3:HUL.InstRepos:dash.current.terms-of-use\#LAA

\section{Share Your Story}

The Harvard community has made this article openly available.

Please share how this access benefits you. Submit a story.

Accessibility 


\title{
3C 220.3: A RADIO GALAXY LENSING A SUBMILLIMETER GALAXY
}

\author{
Martin Haas $^{1}$, Christian Leipski ${ }^{2}$, Peter Barthel $^{3}$, Belinda J. Wilkes ${ }^{4}$, Simona Vegetti $^{5}$, R. Shane Bussmann ${ }^{4}$, \\ S. P. Willner ${ }^{4}$, Christian Westhues ${ }^{1}$, Matthew L. N. Ashby ${ }^{4}$, Rolf Chini ${ }^{1,6}$, David L. Clements ${ }^{7}$, \\ Christopher D. Fassnacht ${ }^{8}$, Assaf Horesh ${ }^{9}$, Ulrich KlaAs ${ }^{2}$, Léon V. E. Koopmans ${ }^{3}$, Joanna Kuraszkiewicz ${ }^{4}$, \\ David J. Lagattuta ${ }^{10,11}$, Klaus Meisenheimer ${ }^{2}$, Daniel Stern ${ }^{12}$, AND Dominika WylezaleK ${ }^{12,13}$ \\ ${ }^{1}$ Astronomisches Institut, Ruhr Universität, Bochum, Germany; haas@astro.rub.de \\ ${ }^{2}$ Max-Planck-Institut für Astronomie, Heidelberg, Germany \\ ${ }^{3}$ Kapteyn Astronomical Institute, University of Groningen, The Netherlands \\ ${ }^{4}$ Harvard-Smithsonian Center for Astrophysics, Cambridge, MA, USA \\ ${ }^{5}$ Max-Planck-Institut für Astrophysik, Garching, Germany \\ ${ }^{6}$ Universidad Catolica del Norte, Antofagasta, Chile \\ ${ }^{7}$ Imperial College, London, UK \\ ${ }^{8}$ University of California, Davis, CA, USA \\ ${ }^{9}$ Division of Physics, Mathematics, and Astronomy, California Institute of Technology, Pasadena, CA, USA \\ ${ }^{10}$ Centre for Astrophysics \& Supercomputing, Swinburne University of Technology, Hawthorn, Australia \\ ${ }^{11}$ ARC Centre of Excellence for All-sky Astrophysics (CAASTRO), 44 Rosehill Street Redfern, NSW 2016, Australia \\ 12 Jet Propulsion Laboratory, California Institute of Technology, Pasadena, CA, USA \\ ${ }^{13}$ European Southern Observatory, Garching, Germany \\ Received 2013 August 23; accepted 2014 June 6; published 2014 July 2
}

\begin{abstract}
Herschel Space Observatory photometry and extensive multiwavelength follow-up have revealed that the powerful radio galaxy (PRG) 3C 220.3 at $z=0.685$ acts as a gravitational lens for a background submillimeter galaxy (SMG) at $z=2.221$. At an observed wavelength of $1 \mathrm{~mm}$, the SMG is lensed into three distinct images. In the observed near infrared, these images are connected by an arc of $\sim 1^{\prime \prime} .8$ radius forming an Einstein half-ring centered near the radio galaxy. In visible light, only the arc is apparent. 3C 220.3 is the only known instance of strong galaxy-scale lensing by a PRG not located in a galaxy cluster and therefore it offers the potential to probe the dark matter content of the radio galaxy host. Lens modeling rejects a single lens, but two lenses centered on the radio galaxy host A and a companion B, separated by $1^{\prime \prime} .5$, provide a fit consistent with all data and reveal faint candidates for the predicted fourth and fifth images. The model does not require an extended common dark matter halo, consistent with the absence of extended bright X-ray emission on our Chandra image. The projected dark matter fractions within the Einstein radii of $\mathrm{A}\left(1^{\prime \prime} .02\right)$ and $\mathrm{B}\left(0^{\prime \prime} .61\right)$ are about $0.4 \pm 0.3$ and $0.55 \pm 0.3$. The mass to $i$-band light ratios of $\mathrm{A}$ and $\mathrm{B}, M / L_{i} \sim 8 \pm 4 M_{\odot} L_{\odot}^{-1}$, appear comparable to those of radio-quiet lensing galaxies at the same redshift in the CfA-Arizona Space Telescope LEns Survey, Lenses Structure and Dynamics, and Strong Lenses in the Legacy Survey samples. The lensed SMG is extremely bright with observed $f(250 \mu \mathrm{m})=440 \mathrm{mJy}$ owing to a magnification factor $\mu \sim 10$. The SMG spectrum shows luminous, narrow C IV $\lambda 1549 \AA$ emission, revealing that the SMG houses a hidden quasar in addition to a violent starburst. Multicolor image reconstruction of the SMG indicates a bipolar morphology of the emitted ultraviolet (UV) light suggestive of cones through which UV light escapes a dust-enshrouded nucleus.
\end{abstract}

Key words: dark matter - galaxies: individual (3C 220.3) - gravitational lensing: strong radio continuum: galaxies - submillimeter: galaxies

Online-only material: color figures

\section{INTRODUCTION}

Powerful radio galaxies (PRGs, with $L_{178 \mathrm{MHz}}>$ $10^{27} \mathrm{~W} \mathrm{~Hz}^{-1} \mathrm{sr}^{-1}$ and Fanaroff-Riley morphology type FR II) mark places of exceptional energy transformation. Giant radio lobes far outside the massive host galaxies are powered by relativistic jets launched from accreting supermassive black holes in the galaxy centers. PRGs are among the most massive galaxies at their epoch, as traced by the $K-z$ brightness-redshift diagram (Lilly \& Longair 1984). In the cold dark matter paradigm of cosmic structure formation (White \& Rees 1978), PRGs might be expected to reside in the most massive dark matter halos and in rich galaxy clusters. For a few PRGs in rich, X-rayluminous clusters, massive amounts of dark matter have been revealed by giant luminous arcs, e.g., 3C 220.1 at $z=0.61$ (Dickinson 1993) or by the weak shear of background galaxies, e.g., 3C 324 at $z=1.2$ (Smail \& Dickinson 1995), both caused by cluster-scale gravitational lensing. However, for PRGs not in a cluster, no galaxy-scale gravitational lensing measurements of their dark matter content have been achieved to date.

This paper reports the first case of a lensing PRG not located in a galaxy cluster. As a bonus, the lensed source is a submillimeter galaxy (SMG). SMGs are dust-enshrouded starforming galaxies at high redshift $(z>1)$ and are thought to be the progenitors of today's massive elliptical galaxies. Lensed examples of SMGs are not rare (Negrello et al. 2010), but a full picture of the population has yet to emerge.

Our discovery of the lensing nature of 3C 220.3 is based on far infrared and submillimeter photometry with the Herschel Space Observatory. The source appeared abnormally bright, suggesting contamination of a background source in the Herschel beam $\left(5^{\prime \prime}-35^{\prime \prime}\right)$. Reprocessed archival Hubble Space Telescope (HST) visible (702 $\mathrm{nm}$ ) images revealed an arc surrounding two objects, indicating that gravitational lensing plays a role. To identify the radio source among the two lensing candidate galaxies, we obtained a deep $3.3 \mathrm{~cm}$ radio map with the Karl 
G. Jansky Very Large Array (JVLA, 0".4 beam width). We performed high resolution imaging at $1.0 \mathrm{~mm}$ with the Submillimeter Array (SMA), near-infrared (NIR, $K^{\prime}$-band, $2.124 \mu \mathrm{m}$ ) adaptive optics (AO) imaging with the $10 \mathrm{~m} \mathrm{Keck}$ II telescope, 3.6 and $4.5 \mu \mathrm{m}$ imaging with the Spitzer Space Telescope, 360-880 nm spectroscopy with the $5 \mathrm{~m}$ Palomar telescope, 320-1000 nm spectroscopy with the $10 \mathrm{~m}$ Keck I telescope, and obtained a $10 \mathrm{ks}$ Chandra X-ray image.

This paper reports results of the imaging and spectroscopy campaigns (Section 2) and of lens modeling (Section 3) to fit the resulting data. Section 4 treats the wavelength dependent morphology of the SMG. Section 5 compares the derived mass to light ratio $(M / L)$ for the PRG to ratios for galaxies at similar redshift, and Section 6 summarizes the results. We adopt a standard $\Lambda$ CDM cosmology $\left(H_{\circ}=72 \mathrm{~km} \mathrm{~s}^{-1} \mathrm{Mpc}^{-1}\right.$, $\Omega_{\Lambda}=0.73$, and $\Omega_{m}=0.27$ ).

\section{OBSERVATIONS AND DATA}

\subsection{Herschel Space Observatory}

Photometric observations of 3C 220.3 were carried out with the Herschel Space Observatory (Pilbratt et al. 2010) using the 70,100 , and $160 \mu \mathrm{m}$ channels of the instrument PACS (Poglitsch et al. 2010) on UT 2011 May 28 in scan-map mode with onsource times of $\sim 80 \mathrm{~s}$ at 70 and $100 \mu \mathrm{m}$ and $\sim 160 \mathrm{~s}$ at $160 \mu \mathrm{m}$. SPIRE (Griffin et al. 2010) observations at 250,350, and $500 \mu \mathrm{m}$ were made on UT 2012 November 5 in small-map mode with $\sim 111$ s on-source time. Data reduction was performed within the Herschel Interactive Processing Environment (HIPE; Ott 2010) following standard procedures including source masking and high-pass filtering. Flux densities were measured for PACS with aperture photometry including corrections for aperture size. SPIRE photometry utilized the timeline source extractor implemented in HIPE. Because the source is bright in all filters, the photometric uncertainties are small, typically a few percent.

3C 220.3 was observed with the MIPS (Rieke et al. 2004) instrument on the Spitzer Space Telescope (Werner et al. 2004) with reported 70 and $160 \mu \mathrm{m}$ flux densities (Cleary et al. 2007) significantly lower than those from Herschel. To understand this discrepancy, we retrieved newly calibrated MIPS maps from the Spitzer archive and performed photometry with aperture corrections from the MIPS Instrument Handbook. ${ }^{14}$ The new 70 and $160 \mu \mathrm{m}$ Spitzer photometry yielded higher flux densities which now are consistent within $15 \%$ with the Herschel results. The Spitzer MIPS $24 \mu \mathrm{m}$ photometry agrees to within $4 \%$ with $22 \mu \mathrm{m}$ photometry from the Wide-field Infrared Survey Explorer (WISE; Wright et al. 2010).

For comparison with 3C 220.3, a sample of 12 PRGs was taken from the 3CR catalog. The sample (C. Westhues et al., in preparation) consists of galaxies with $0.6<z<0.8$ and radio and mid-infrared properties similar to those of 3C 220.3. The MIPS $24 \mu \mathrm{m}$ photometry of galaxies in the comparison sample is consistent with the WISE data.

\subsection{Karl G. Jansky Very Large Array}

The deepest radio image of $3 \mathrm{C} 220.3$ to date, at $\lambda=6 \mathrm{~cm}$ by Mullin et al. (2006), shows the double-lobed morphology but not the unresolved core. With the aim of detecting the core, we observed 3C 220.3 with the Karl G. JVLA in its long-baseline A configuration on UT 2012 November 30 employing 25 antennas.

\footnotetext{
14 http://irsa.ipac.caltech.edu/data/SPITZER/docs/mips/ mipsinstrumenthandbook/50
}

Table 1

Photometry of 3C 220.3

\begin{tabular}{lc}
\hline \hline $\begin{array}{l}\text { Observed Wavelength } \\
(\mu \mathrm{m})\end{array}$ & $\begin{array}{c}\text { Flux Density } \\
(\mathrm{mJy})\end{array}$ \\
\hline 0.702 & $0.0253 \pm 0.00$ \\
2.124 & $0.193 \pm 0.03$ \\
3.6 & $0.275 \pm 0.028$ \\
4.5 & $0.283 \pm 0.028$ \\
70. & $29.5 \pm 5$ \\
100. & $102 \pm 7$ \\
160. & $289 \pm 9$ \\
250. & $440 \pm 15$ \\
350. & $403 \pm 20$ \\
500. & $268 \pm 30$ \\
1000. & $51 \pm 12$ \\
\hline
\end{tabular}

Note. Sum of all components A, B, knots, and arc.

The bandwidth was $2048 \mathrm{MHz}$ centered at $9 \mathrm{GHz}$ (X band). Prime flux calibrators were 3C 48 and 3C 147, and the nearby radio source $\mathrm{J} 1010+8250$ served as phase calibrator. The total on-source integration time was 37 minutes.

The resulting image, which was made using CASA and employed natural weighting of the visibility data, has an angular resolution of $\sim 0^{\prime \prime}$. 4 . It shows the $\sim 9^{\prime \prime}$ double-lobed morphology reported previously (Mullin et al. 2006) and an unresolved core with a flux density of $0.8 \mathrm{mJy}$ (Figure 1 ).

\subsection{Hubble Space Telescope}

The HST archive contains two images of 3C 220.3 with WFPC2 in the broadband filter F702W taken on 1994 March 11 and 1995 May 19, each with an exposure time of $300 \mathrm{~s}$. The reprocessed preview images now in the archive ${ }^{15}$ clearly show an arc, which was not visible on the early map published by McCarthy et al. (1997). ${ }^{16}$ To improve the contrast further, we have retrieved, aligned, and co-added the two F702W images (Figure 1), adjusting the positions of A and B to match those from the Keck AO (see Section 2.4).

Photometric calibration was derived by measuring the total $702 \mathrm{~nm}$ flux density in a circular aperture of 5."6 diameter, containing sources $\mathrm{A}$ and $\mathrm{B}$ and the entire arc, and scaling it to the total $r$ - and $i$-band photometry of 3C 220.3 listed by the Sloan Digital Sky Survey (SDSS, Data Release 9). ${ }^{17}$ The total magnitudes (in the AB system) are $r=20.96 \pm 0.13$ at $623 \mathrm{~nm}$ and $i=20.09 \pm 0.10$ at $764 \mathrm{~nm}$, yielding the total $702 \mathrm{~nm}$ flux density listed in Table 1 . The $702 \mathrm{~nm}$ flux densities of A and B separately are listed in Table 2.

\subsection{Near-infrared Imaging with Keck Adaptive Optics}

The 3C 220.3 system was observed with the Keck II telescope on UT 2012 December 24 utilizing NIRC2 behind the AO bench. The laser guide star system was used in conjunction with an

\footnotetext{
15 http://archive.stsci.edu/

16 McCarthy et al. (1997), reporting on early HST imaging of 3C radio galaxies, mentioned the possible association of 3C 220.3 with a gravitational arc, citing "M. Dickinson, private communication." While that claim now fortuitously turns out to have been correct, the comment was at the time meant to apply to 3C 220.1. Dickinson has further confirmed that the ground-based imaging of 3C 220.3 available to him at that time was quite poor, and 3C 220.3 was one of the few targets omitted from his comprehensive $3 \mathrm{C}$ optical imaging survey (Dickinson 1994). The 3C 220.1 lensing arc is $9^{\prime \prime}$ in radius, but the lens is an entire cluster (Ota et al. 2000; Comerford et al. 2006; Belsole et al. 2007).

17 http://www.sdss3.org/dr9/
} 

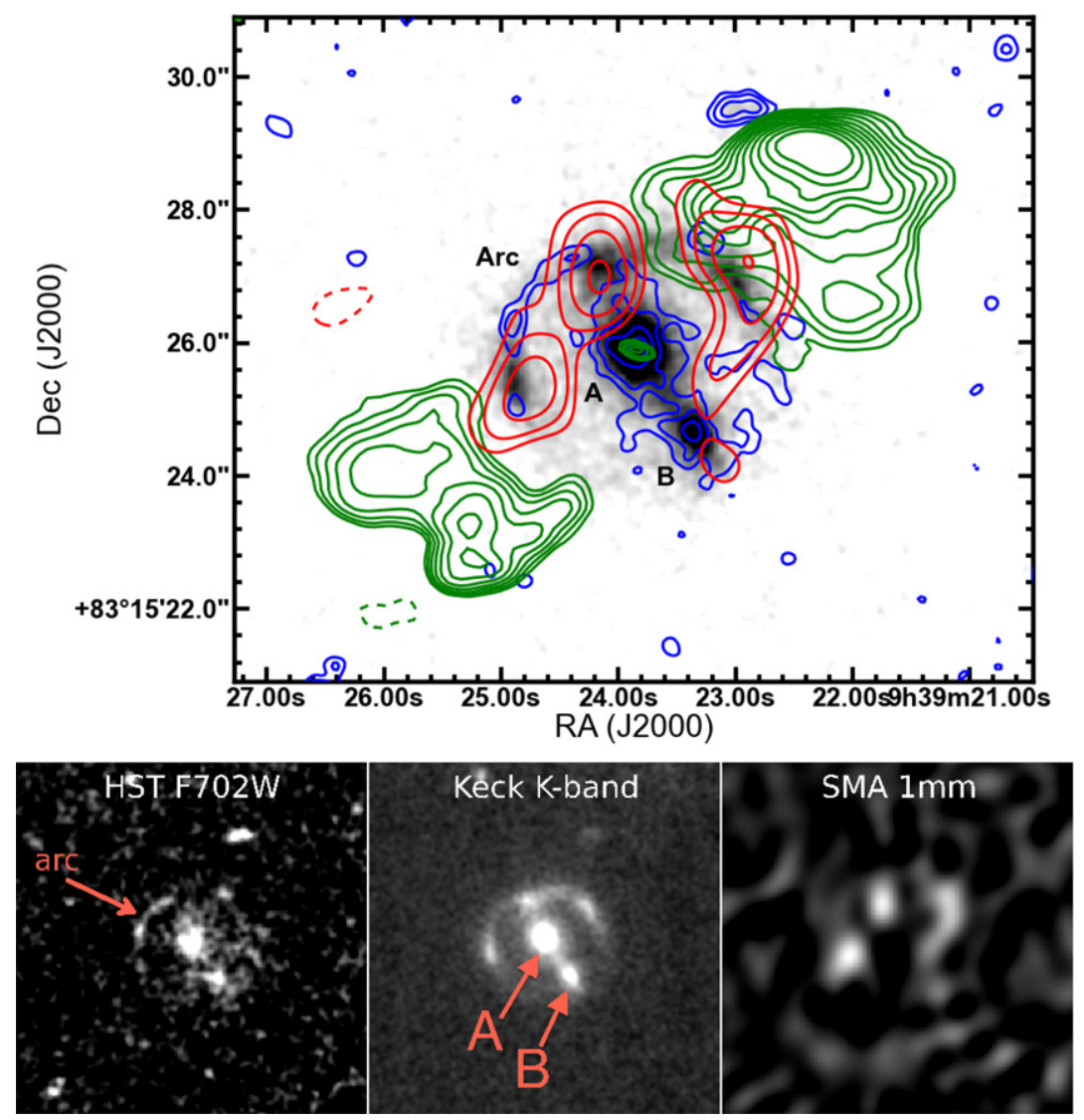

Figure 1. Top: negative gray-scale Keck AO ( $K^{\prime}$-band, $\left.2.124 \mu \mathrm{m}\right)$ image of $3 \mathrm{C} 220.3$ with linear contours at other wavelengths: blue $=H S T$ at $702 \mathrm{~nm}$; red $=\mathrm{SMA}$ at $1 \mathrm{~mm}$; green = JVLA at $3.3 \mathrm{~cm}$. The position of the radio core is at R.A. = 09:39:23.837, decl. = +83:15:25.90 (J2000). Bottom: high resolution HST, Keck AO, and SMA images of 3C 220.3 with key components labeled. Image sizes are 12" square with north up and east to the left.

(A color version of this figure is available in the online journal.)

Table 2

Photometry of 3C 220.3 A and B and Parameters of the Surface Profile Modeling

\begin{tabular}{|c|c|c|c|c|c|c|}
\hline Lens & $702 \mathrm{~nm}$ & $2.124 \mu \mathrm{m}$ & Sérsic Index $n$ & $\begin{array}{c}r_{\text {eff }} \\
(\operatorname{arcsec})\end{array}$ & Axis Ratio $b / a$ & $\begin{array}{l}\text { P.A. } \\
\text { (deg) }\end{array}$ \\
\hline $\mathrm{A}$ & $14.8 \pm 3.5$ & $76.7 \pm 15.0$ & $0.9 \pm 0.09$ & $0.29 \pm 0.04$ & $0.7 \pm 0.07$ & $29 \pm 12$ \\
\hline B & $4.45 \pm 1.1$ & $20.0 \pm 3.0$ & $1.0 \pm 0.12$ & $0.20 \pm 0.04$ & $0.6 \pm 0.11$ & $31 \pm 25$ \\
\hline
\end{tabular}

Notes. The flux densities in $\mu \mathrm{Jy}$ were determined using SExtractor with auto aperture option. The resulting elliptical apertures have an average circular diameter of about twice the Einstein radii of A and B. For surface profile modeling, the Keck AO data were fitted by PSF-convolved Sérsic profiles.

$R=15.5$ mag tip-tilt star located $23^{\prime \prime}$ from 3C 220.3. The wide camera was used, which provides a pixel scale of 0.0397 and a field of view of $40^{\prime \prime}$ on a side. We obtained nine dithered exposures in the $K^{\prime}$ band $(2.124 \mu \mathrm{m})$, each exposure consisting of four co-added frames of $30 \mathrm{~s}$ each.

The data were reduced using a Python pipeline that flat-fields the data, subtracts sky emission, aligns the images, and drizzles to create the final image. The last step corrects for distortions that create a non-uniform pixel scale. The final image (Figure 1) used eight of the nine input images, giving a total exposure time of $960 \mathrm{~s}$. The image center position was derived from three Two Micron All Sky Survey (2MASS) point sources in the frame. With this astrometry, A is within 0'.4 of the radio core position, and the final image coordinates were adjusted by this amount to match $\mathrm{A}$ to the radio core position (Figure 1).

Photometric calibration was derived using an object (probably a compact galaxy) located on the Keck AO mosaic at
R.A. $=09: 39: 34.18$, decl. $=+83: 14: 51.4(\mathrm{~J} 2000)$, and $K_{s}=$ $15.04 \pm 0.124$ mag listed by 2MASS.

\subsection{Spitzer/IRAC Imaging}

We observed 3C 220.3 on UT 2013 June 14 using the IRAC instrument (Fazio et al. 2004) of the Spitzer Space Telescope (Werner et al. 2004). Nine dithered exposures of $30 \mathrm{~s}$ each were secured at $3.6 \mu \mathrm{m}$ and at $4.5 \mu \mathrm{m}$. The dithered images were combined and resampled to a pixel size of 0!3 using IRACproc (Schuster et al. 2006) and MOPEX.

3C 220.3 is clearly resolved at both 3.6 and $4.5 \mu \mathrm{m}$. Approximate deconvolution gives full widths at half maximum surface brightness of $3{ }^{\prime \prime} 0$ at $3.6 \mu \mathrm{m}$ and 3." 4 at $4.5 \mu \mathrm{m}$. These are comparable to the diameter of the Einstein ring (3".6). However, the limited angular resolution makes it not straightforward to distinguish the components seen on the KECK AO image, i.e., 
sources $\mathrm{A}$ and $\mathrm{B}$ and the knots and arc. The IRAC photometry of 3C 220.3 listed in Table 1 is consistent with that from WISE.

\subsection{Submillimeter Array}

SMA imaging of 3C 220.3 was obtained with Director's Discretionary Time in the 2012B semester as part of program 2012B-S084. Observations were conducted in extended array configuration (maximum baseline length of $226 \mathrm{~m}$ ) on UT 2013 January 11 in good weather conditions $\left(\tau_{225 \mathrm{GHz}} \sim 0.08\right)$ for $t_{\text {int }}=9.6 \mathrm{hr}$ on-source integration time. Compact array observations (maximum baseline length of $76 \mathrm{~m}$ ) were obtained on UT 2013 February 10, again in good weather conditions $\left(\tau_{225 \mathrm{GHz}} \sim 0.08\right)$ for $t_{\text {int }}=6.7 \mathrm{hr}$. Phase stability for both observing nights was good (phase errors between $10^{\circ}$ and $20^{\circ} \mathrm{rms}$ ). The SMA receivers provided $8 \mathrm{GHz}$ of instantaneous bandwidth (considering both sidebands) and were tuned such that the upper sideband was centered on $302.927 \mathrm{GHz}$.

Calibration of the $u v$ visibilities was performed using the Interactive Data Language MIR package. The blazar 3C 84 was used as the primary bandpass calibrator and Titan for absolute flux calibration. The nearby quasars $0721+713\left(S_{303 \mathrm{GHz}}=\right.$ $5.5 \mathrm{Jy}, 13.6$ from target $)$ and $1048+717\left(S_{303 \mathrm{GHz}}=0.8 \mathrm{Jy}\right.$ 12.0 from target) were used for phase and gain calibration. We checked the reliability of the phase-gain solutions using the quasar $1044+809\left(S_{303 \mathrm{GHz}}=0.3 \mathrm{Jy}, 3.2\right.$ from target $)$.

The Multichannel Image Reconstruction, Image Analysis, and Display (MIRIAD) software package (Sault et al. 1995) was used to invert the $u v$ visibilities and deconvolve the dirty map. Natural weighting was chosen to obtain maximum sensitivity and resulted in an elliptical Gaussian beam with a full-width half-maximum (FWHM) of $1^{\prime \prime} .40 \times 1^{\prime \prime}$. 17 and major axis position angle $146^{\circ}$ east of north.

\subsection{Visible Spectroscopy}

\subsubsection{Palomar}

We observed 3C 220.3 for $1 \mathrm{hr}$ using the Double Spectrograph on the Palomar $5 \mathrm{~m}$ Hale Telescope on UT 2013 February 12. The observations were obtained with three $1200 \mathrm{~s}$ exposures at a position angle of $31^{\circ}$ and the $5500 \AA$ dichroic, providing a wavelength coverage of 3200-8800 $\AA$. Conditions were photometric, though the seeing was poor $\left(>2^{\prime \prime}\right)$. The observations used the $1^{\prime \prime} .5$ wide longslit, the $600 / 4000$ blue grating (resolving power $R \sim 1000$ ), and the 600/10000 red grating ( $R \sim 2400)$. We processed the data using standard techniques and flux calibrated the spectra using standard stars from Massey \& Gronwall (1990) observed during the same night.

The Palomar observations failed to detect the arc but confirmed the redshift of $3 \mathrm{C} 220.3$ at $z=0.685$ based on the detection of narrow $\left.\mathrm{C}_{\mathrm{II}}\right] \lambda 2326$, [O II] $\lambda 3727$, and [O III] $\lambda 5007$ emission lines, typical of PRGs (McCarthy 1993; Stern et al. 1999). The only previously published redshift of 3C 220.3 to our knowledge was based on [O II] $\lambda 3727$ and [Ne v] $\lambda 3346$ emission with no figure available (Spinrad et al. 1985). ${ }^{18}[\mathrm{Ne} v]$ (redshifted to $\sim 5560 \AA$ ) was not detected in our Palomar spectrum, likely owing to its proximity to the dichroic crossover.

\footnotetext{
18 Jasper Wall kindly showed us a plot of another spectrum of 3C 220.3 taken with the $4 \mathrm{~m}$ William Herschel Telescope, La Palma, yielding $z=0.685$ based on a single emission line adopted to be $[\mathrm{O}$ II $] \lambda 3727$. This spectrum is mentioned at http://www-astro.physics.ox.ac.uk/ sr/grimes.html.
}

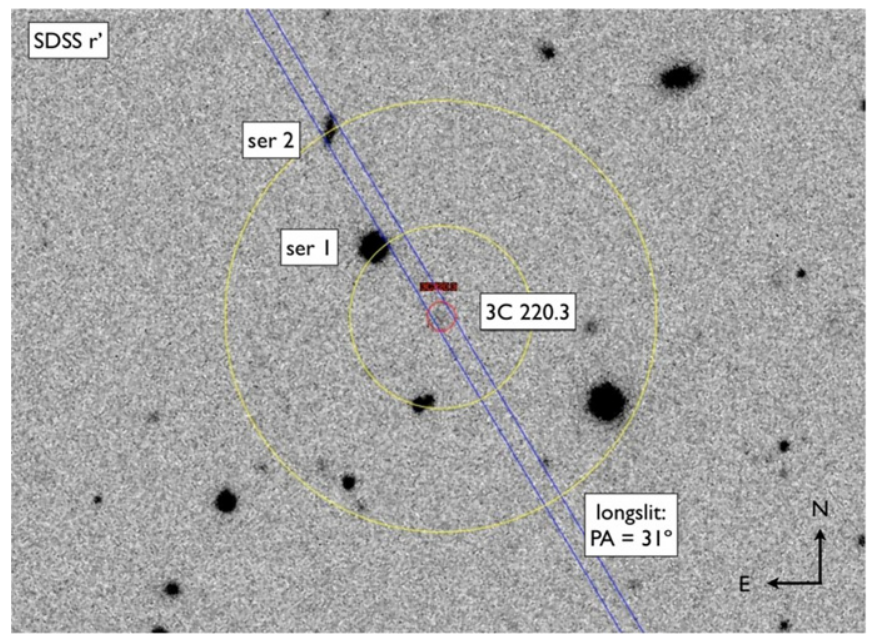

Figure 2. Keck LRIS slit position (blue lines) overlaid on an SDSS $r$-band negative image of the $3 \mathrm{C} 220.3$ system. The small red circle marks the location of $3 \mathrm{C} 220.3$, and the larger yellow circles (radii $19^{\prime \prime}$ and $45^{\prime \prime}$ ) go through two serendipitously hit sources labeled "ser 1" and "ser 2."

(A color version of this figure is available in the online journal.)

\subsubsection{Keck}

With the goal of measuring the arc redshift, we performed additional spectroscopy of 3C 220.3 with the Low Resolution Imaging Spectrometer (LRIS; Oke et al. 1995) at the Cassegrain focus of the Keck I telescope. We obtained three $1200 \mathrm{~s}$ observations at a position angle of $31^{\circ}$. The observations used the $1^{\prime \prime} .0$ wide longslit, the $5600 \AA$ dichroic, the $400 / 3400$ blue grism $(R \sim 900)$, and the $400 / 8500$ red grating $(R \sim 1400)$. The position of the slit is illustrated in Figure 2. Conditions were photometric with $\sim 1^{\prime \prime} .5$ seeing. We processed the data using standard techniques and flux calibrated the spectra using observations of G191-B2B, a standard star from Massey \& Gronwall (1990), obtained during the same night.

The Keck spectrum confirms the redshift of the radio galaxy, detecting the emission lines previously seen in the Palomar spectrum as well as weak, narrow [Mg II] $\lambda 2800$ and $\mathrm{H} \beta$ emission (Figure 3). More excitingly, the Keck spectrum reveals two additional narrow emission lines with a slight spatial offset from the primary continuum. These lines are associated with the arc (Figure 4), and we identify the lines as C IV $\lambda 1549$ and He II $\lambda 1640$ at redshift $z=2.221$. These lines are typical of radio galaxies and type- 2 active galactic nucleus (AGN), and the high C Iv luminosity $\left(\sim 2 \times 10^{42} \mathrm{erg} \mathrm{s}^{-1}\right)$ argues for a luminous AGN, i.e., a QSO (e.g., Stern et al. 2002).

In an odd coincidence, Ly $\alpha$ for the lensed background source is shifted to observed $3916 \AA$, which coincides with $\left.\mathrm{C}_{\text {II] }}\right] \lambda 2326$ from the radio galaxy. The spatial location of this emission in the two-dimensional spectrum (Figure 4) suggests that the emission at this wavelength predominantly coincides with the radio galaxy; Ly $\alpha$ emission from the lensed, background SMG appears narrow and weak (e.g., relative to the C IV line for an average type-2 AGN), likely due to absorption and/or resonance scattering by the dust- and gas-rich SMG.

Examining the Keck spectra to uncover the redshift of source B provides a less certain result. The higher spectral dispersion red-arm data were binned spatially to 0'.27 per pixel compared to $0{ }^{\prime} .135$ per pixel for the blue arm. Based on both the [O II] $\lambda 3727$ and the $\mathrm{H} \beta$ emission lines, it appears that source $\mathrm{B}$ is at nearly the same redshift as source A but blue-shifted by $\sim 5 \AA$ (4 pixels). 


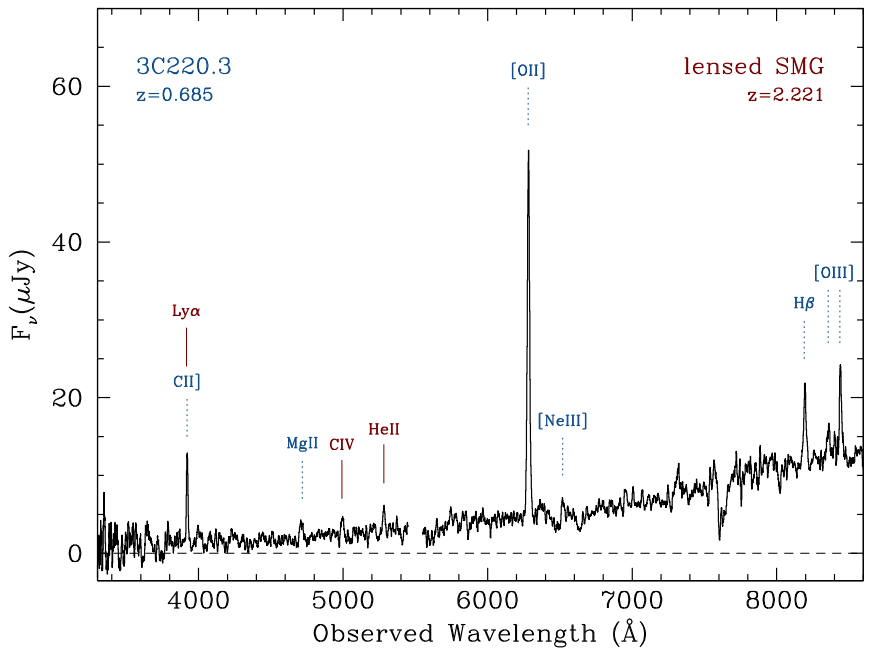

Figure 3. Keck LRIS spectrum of 3C 220.3 system with prominent lines labeled. Blue dotted lines label emission lines associated with the radio galaxy at $z=0.685$, while red solid lines label emission lines associated with the lensed SMG at $z=2.221$. The feature at $3921 \AA$ is associated with emission lines from both sources. The gap at $5500 \AA$ corresponds to the dichroic separating the blue and red arms of LRIS. Absorption at $7600 \AA$ is due to the uncorrected atmospheric $A$ band.

(A color version of this figure is available in the online journal.)

\subsection{Chandra X-Ray Observations}

3C 220.3 was observed with Chandra ACIS-S on UT 2013 January 21 for $10 \mathrm{ks}$. The total, broadband $(0.3-\mathrm{keV}) \mathrm{X}$-ray flux density of the system is $\sim 1.14 \times 10^{-14} \mathrm{erg} \mathrm{cm}^{-2} \mathrm{~s}^{-1}$, which yields a luminosity of $L_{X}=2.4 \times 10^{43} \mathrm{erg} \mathrm{s}^{-1}$ if all photons originate at $z=0.685$. The detection includes 16 photons (Figure 5) with 1.8 expected from a smooth background. Photons are distributed across the system. It is not generally possible to identify the X-ray photons with specific structure(s), although two photons are aligned with the radio core (implying $L_{X} \sim 3 \times 10^{42} \mathrm{erg} \mathrm{s}^{-1}$ ). The observational upper limit of $\sim 10^{43} \mathrm{erg} \mathrm{s}^{-1}$ for extended, hot X-ray emitting gas is about a factor of 10 below typical values for X-ray clusters (Pratt et al. 2009).

\subsection{Spectral Energy Distribution}

Figure 6 and Table 1 give the spectral energy distribution (SED) of the combined objects. The Herschel beam includes all flux from the SMG knots and arc and from the two lenses A and $\mathrm{B}$, but the contributions of $\mathrm{A}$ and $\mathrm{B}$ are likely to be negligible. In the comparison sample of 12 Herschel observed $3 \mathrm{C}$ objects,

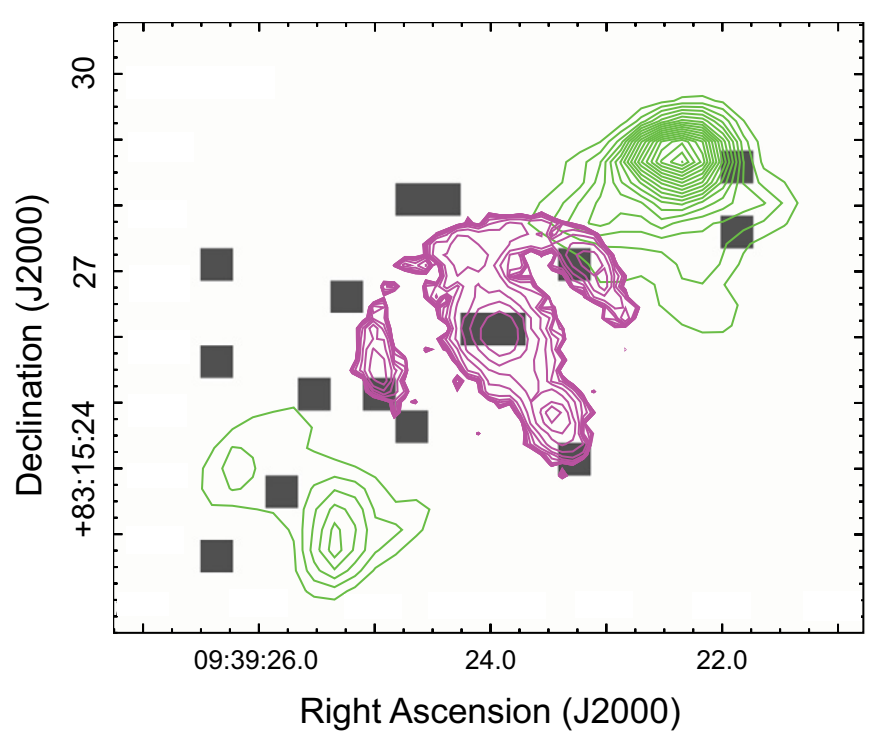

Figure 5. Chandra X-ray photon image (black squares) toward 3C 220.3, with Keck $K^{\prime}$-band contours (violet) and $6 \mathrm{~cm}$ radio contours (green; from Mullin et al. 2006).

(A color version of this figure is available in the online journal.)

only three were detected with Herschel. Their SEDs peak at $\lambda_{\text {obs }} \sim 100 \mu \mathrm{m}$ with flux densities below $100 \mathrm{mJy}$ and decline toward shorter and longer wavelengths. The remaining nine $3 \mathrm{C}$ radio galaxies are undetected at $70-160 \mu \mathrm{m}$ with upper limits well below the 3C 220.3 flux densities. Lens galaxy B is even less likely than A to contribute to the FIR flux density because it is $\sim 3$ times fainter than $\mathrm{A}$ in the NIR and has a slightly bluer visible-NIR color (Table 2, $f(2.124 \mu \mathrm{m}) / f(702 \mathrm{~nm}) \sim 5$ and 4 for A and B, respectively). The small 70-160 $\mu \mathrm{m}$ flux densities of the 12 comparison $3 \mathrm{C}$ objects and the non-detection of $\mathrm{A}$ and $\mathrm{B}$ at $1 \mathrm{~mm}$ imply that most of the $\lambda>200 \mu \mathrm{m}$ flux comes from the SMG at $z=2.221$.

The visible-to- $850 \mu \mathrm{m}$ flux density ratio of the $3 \mathrm{C} 220.3 \mathrm{SMG}$ is consistent with the median of 73 SMGs at $z \sim 2.2$ (Chapman et al. 2005; Figure 6). The best-fit dust temperature $T \sim 36 \mathrm{~K}$ of the 250-1000 $\mu \mathrm{m}$ dust emission (for a dust emissivity index $\beta=1.5$ and $z=2.221$ ) is typical for unlensed SMGs (Magnelli et al. 2012). ${ }^{19}$ If the dust were associated with the radio source at $z=0.685$, its temperature would be $<20 \mathrm{~K}$ and its dust mass, $M_{d}>10^{11} M_{\odot}$, would be similar to the stellar mass, cooler

19 SED fitting with several dust components covering a range of temperatures does not result in a unique stable solution, but the temperature $T$ of the FIR-millimeter decline is a quite stable, widely used characteristic parameter.

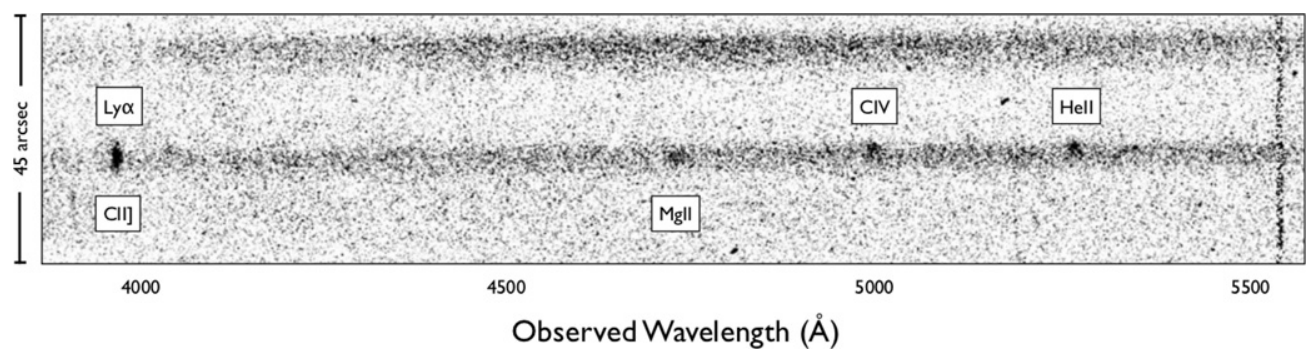

Figure 4. Negative image of the blue side of the Keck LRIS spectrum of 3C 220.3. The horizontal and vertical axes represent the spectral and spatial dimensions, respectively. The horizontal dark stripe in the middle of the frame corresponds to the $3 \mathrm{C} 220.3$ system, and the upper dark stripe originates from the faint extended source labeled "ser 1" in Figure 2. The 3C 220.3 stripe has a width of $\sim 4$ ", the lower part originating from source B, the central part from source A, and the upper part from the arc. The dark knots in the stripe are due to emission lines as labeled. Mg II $\lambda 2798$ from the radio galaxy (source A) is centrally located in the continuum, while the C IV and He II lines are offset upward, indicating an origin in the arc. The line on the left side (at $3920 \AA$ ) seems more central, suggesting it is primarily C II from the radio galaxy rather than Ly $\alpha$ from the lensed SMG. 


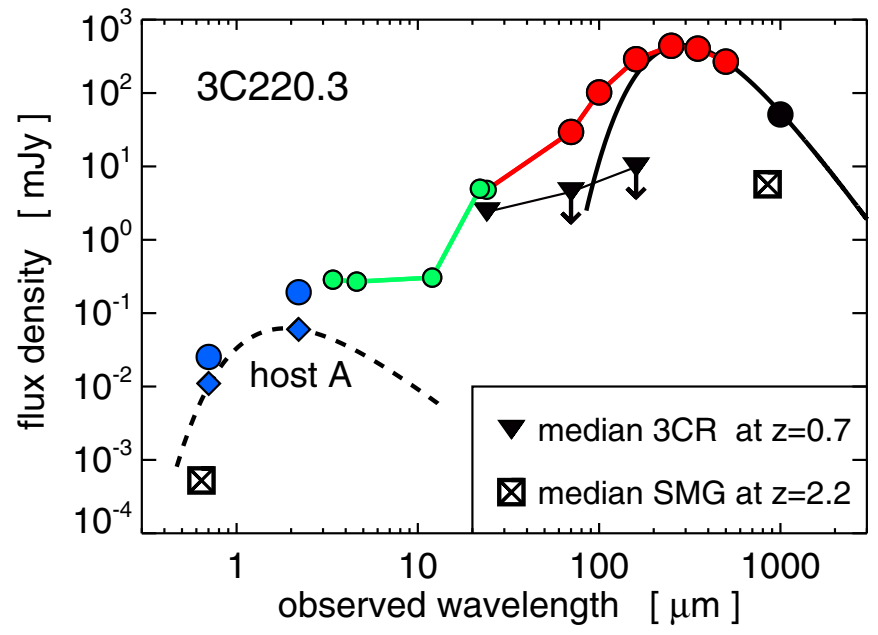

Figure 6. Spectral energy distribution of the 3C 220.3 system. Filled circles show flux densities from the entire source: large red (Herschel), small green (WISE and Spitzer), large black (SMA), large blue (HST and Keck). A solid line shows a graybody fit through the SMA and $\geqslant 250 \mu \mathrm{m}$ Herschel data points $(T=36 \mathrm{~K}$ at $z=2.221$, dust emissivity index $\beta=1.5)$. Blue diamonds show the HST and Keck flux densities of lens A (defined in Figure 1) connected by a blackbody (dashed line). Triangles show the median 24-160 $\mu$ m flux densities of twelve 3C radio galaxies at $z \sim 0.7$. X-squares show the median $R$-band and $850 \mu \mathrm{m}$ flux densities of 73 SMGs at $z \sim 2.2$ (Chapman et al. 2005).

(A color version of this figure is available in the online journal.)

and more massive than typically observed in AGN ( $>30 \mathrm{~K}$, $\left.M_{d}<0.01 M_{\text {stars }}\right)$. Accounting for a total magnification factor $\mu \sim 10$ derived from lens modeling (Section 3), the intrinsic (demagnified) FIR luminosity of the SMG is $L($ FIR $) \sim 1 \times 10^{13} L_{\odot}$.

\section{LENS MODELING}

To derive the lens model of the 3C 220.3 system, we used the Keck AO image because it has higher signal-to-noise ratio $(\mathrm{S} / \mathrm{N})$ and shows more details than the HST and SMA images (Figure 1). The lens parameters derived from the Keck image were then used to model the HST and SMA images.

We first tried a model with a single lens centered on galaxy A, assuming object B is part of the lensed source. This failed because it leaves bright residuals at the $\mathrm{B}$ position. In other words, B is too bright relative to the other lensed features in the Keck image to be fully explained as part of the lensed image. Moreover, B is not seen in the SMA image. The (hypothetical) case that $\mathrm{B}$ is a low-luminosity foreground galaxy with mass too low to influence the lensing leaves bright residuals at the $\mathrm{B}$ position. The remaining possibility is that $\mathrm{B}$ is part of the lens. Therefore, our preferred model has two lenses centered on galaxies $\mathrm{A}$ and $\mathrm{B}$.

\subsection{Keck AO Image}

Lens models were derived from a fully Bayesian, grid-based modeling technique (Koopmans 2005; Vegetti \& Koopmans 2009) that simultaneously solves for the parameters $\eta$ of the lensing galaxies and the surface brightness distribution of the background source $\mathbf{s}$. The Bayesian prior was expressed as the source regularization form $\mathbf{R}$ with regularization level $\lambda$ (Vegetti $\&$ Koopmans 2009). Because this prior is flat in $\log (\lambda)$ and $\eta$, the maximum of $P(\mathbf{d} \mid \lambda, \eta, \mathbf{M}, \mathbf{R})$, that is, the probability of the data $\mathbf{d}$ given a lens mass model $\mathbf{M}$, determines the most probable a posteriori fit (see Equation (35) of Vegetti \& Koopmans 2009). The Bayesian approach takes into account the smoothness of the source via the regularization $\mathbf{R}$ at the level $\lambda$ (Suyu et al. 2006).
For model fitting we have used the uncertainty map computed as the quadrature sum of the image read out noise and Poisson noise using the number of detected photons. Notably, the fitting results are very similar (within a few percent) to those obtained when using a uniform uncertainty determined from clean background areas of the image.

To avoid that the model is affected by image pixels unrelated to the source, all model fitting was restricted to the "smallestpossible" mask as shown in Figure 7 . The mask was set by smoothing the images, then making an $\mathrm{S} / \mathrm{N}$ cut. This ensures that structure, that is present but below the noise level, is not cut off from the data. Thus, the mask is a compromise between being large enough to allow identification of possible extended residuals and being small enough to cover only the arc and the area around $\mathrm{B}$, where the fourth counter-image is expected.

We here consider three scenarios. In case 1 , object $B$ is part of the lensed source; in case 2, B is a low-luminosity foreground galaxy with mass too small to influence the lensing; and in case 3 , B is part of the lens. The lens modeling began with case 1 , using a postage stamp image centered on the lens system but with the lensing galaxy (A) subtracted using analytic models. For cases 2 and 3 both galaxies (A and B) were subtracted. The surface brightness of the galaxies $\mathrm{A}$ and $\mathrm{B}$ is best fitted by pointspread function (PSF)-convolved Sérsic $(n \sim 1)$ profiles (and not by de Vaucouleurs profiles). Their parameters are listed in Table 2 .

The lens mass distribution was modeled as an ellipsoid with a free power-law slope for the density profile. In general, such approximations provide good fits to describe the combined luminous and dark mass profiles (e.g., Treu \& Koopmans 2004; Auger et al. 2009). The profiles were centered on the light distribution of A (or A and B), allowing for a small positional deviation. The parameters of the best-fit single and double lens models are listed in Table 3.

Case 1. Figure 7 shows that the positions of the three knots connected by the arc can roughly be fitted using the single lens A alone. However, the image residual (data minus model) contains not only a bright relict at the position of B but also negative features of the three knots and the arc (seen as blue arcs in the bottom left panel of Figure 7). In other words, B is too bright relative to the other lensed features in the Keck image to be fully explained as part of the lensed image. In addition, the light profile of B is well fit by a smooth Sérsic profile that is unlike the complex structure of the three images along the arc, and finally the model requires the lensed source galaxy to be highly distorted. Overall case 1 is a poor fit to the data, and this is confirmed by the Bayesian evidence (Table 3).

Case 2. Figure 8 shows that the three knots connected by the arc can be fitted, but there is a bright negative residual just at the $\mathrm{B}$ position because this model requires a counter-image of the arc having about $2 / 3$ the flux of the three bright knots. Therefore, B should be seen in the SMA image, but it is not (Figures 1 and 10). These discrepancies lead us to reject the single-lens model and consider B as part of the lens, consistent with its probable redshift (Section 2.7.2).

Case 3. Using two lenses significantly improves the modeling results. ${ }^{20}$ Figure 9 illustrates the consistency of the observed and

\footnotetext{
20 The two-lens model includes the possibility that B is a lens but not necessarily at the same redshift as A, because any two-lens-plane system can be modeled to first order as a single lens plane with a rescaled Einstein radius (Keeton 2001). The Einstein radii are free parameters of the model, and therefore our results are independent of the true redshift of $\mathrm{B}$. If its redshift is lower than assumed, its mass has to be higher, but all other model results are exactly the same.
} 

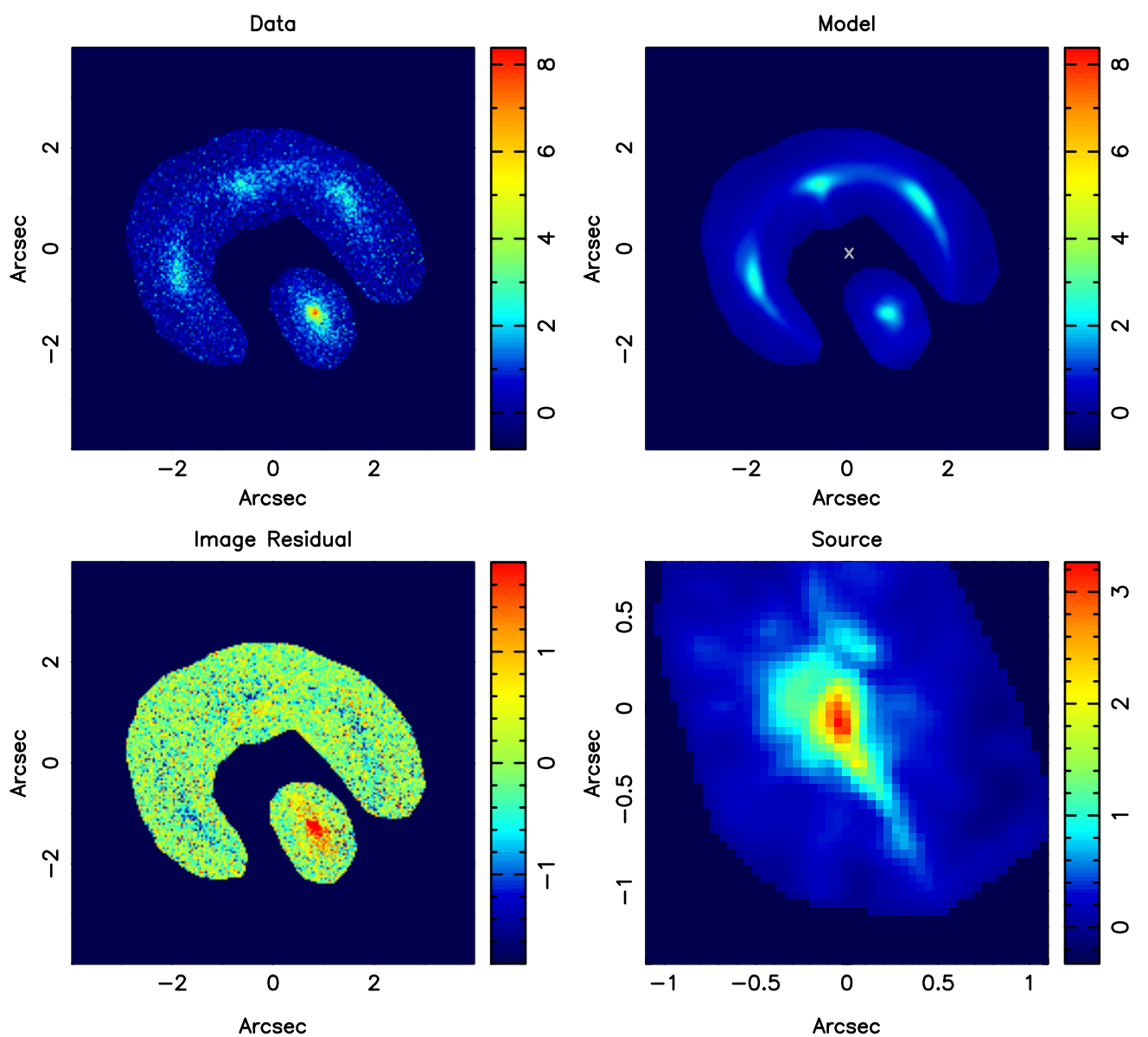

Figure 7. Results from modeling the Keck AO image with a single lens centered on galaxy A, located at $(0,0)$. The regions outside the Einstein half ring and source B are masked and not used for the model fit. While the total map has $200 \times 200=40,000$ pixels, the area used for modeling has 10,912 pixels. North is up and east is to the left. Top left: data after subtraction of A. Top right: modeled image. The cross marks the position of A. Bottom left: residual image, data minus model image. Bottom right: reconstructed image of the source in the source plane $(50 \times 50=2500$ source pixels $)$.

(A color version of this figure is available in the online journal.)

Table 3

Model Parameters from the Keck AO Image

\begin{tabular}{|c|c|c|c|c|c|c|c|c|c|}
\hline (1) & (2) & (3) & (4) & (5) & (6) & (7) & (8) & (9) & (10) \\
\hline Lens & $\begin{array}{c}\text { Einstein } \\
\text { Radius (") }\end{array}$ & $\begin{array}{l}\text { Lens Position } \\
\quad x, y\left({ }^{\prime \prime}\right)\end{array}$ & $b / a$ & $\begin{array}{l}\text { P.A. } \\
\text { (deg) }\end{array}$ & $\begin{array}{l}\text { Slope } \\
\text { (s) }\end{array}$ & $\begin{array}{l}\text { Shear } \\
\text { Strength }\end{array}$ & $\begin{array}{c}\text { Shear } \\
\text { P.A. (deg) }\end{array}$ & $\begin{array}{c}\text { Lens Mass } \\
10^{11} M_{\odot}\end{array}$ & $\ln E v$ \\
\hline Single lens, case 1 & & & & & & & & & -10341 \\
\hline A & 1.67 & $0.043,-0.183$ & 0.61 & 38.7 & 2.071 & 0.093 & 5.4 & 8.9 & \\
\hline Unc A & 0.09 & $0.007,0.010$ & 0.10 & 8 & 0.140 & 0.031 & 20 & 1.1 & \\
\hline Single lens, case 2 & & & & & & & & & -9660 \\
\hline A & 1.68 & $0.113,-0.324$ & 0.27 & 30.8 & 2.310 & 0.146 & 13.3 & 9.0 & \\
\hline Unc A & 0.08 & $0.013,0.028$ & 0.16 & 12 & 0.230 & 0.029 & 24 & 1.0 & \\
\hline Double lens, case 3 & & & & & & & & & -9409 \\
\hline A & 1.02 & $-0.174,0.223$ & 0.61 & 35.7 & 1.837 & 0.098 & 5.0 & 3.5 & \\
\hline Unc A & 0.07 & $0.010,0.010$ & 0.13 & 10 & 0.090 & 0.030 & 23 & 0.5 & \\
\hline B & 0.61 & $0.861,-1.415$ & 0.74 & 42.1 & 1.964 & & & 1.2 & \\
\hline Unc B & 0.05 & $0.007,0.008$ & 0.12 & 15 & 0.150 & & & 0.2 & \\
\hline
\end{tabular}

Notes. The uncertainties (labeled "unc") are listed in the line underneath the parameter values. The lens positions (in Column 3) are relative to the axis from the observer to the source. Columns 4 and 5 give the axis ratio $\left(b / a=\right.$ minor/major axis) and position angle of the lenses. Radial mass surface density $\rho \propto r^{-s}$ (in Column 6), a slope $s=2.0$ corresponds to a singular isothermal ellipsoid. Column 10 gives the natural logarithm of the Bayesian evidence (ln $E v$ ) of cases 1-3, respectively.

modeled images and the featureless image residual all along the arc. The two-lens model predicts two additional faint images: a fourth image on the opposite side of the Einstein half-ring and a fifth (central) image. Their positions agree with two faint blobs in the Keck data after subtraction of a galaxy model for
A and B and with faint features in the SMA map (Figure 10). However, both images are close to the noise level and need to be confirmed with deeper optical/NIR data and a higher spatial resolution $1 \mathrm{~mm}$ map. The magnification factor of the two-lens model at $2.124 \mu \mathrm{m}$ is $\mu \sim 7 \pm 2$. 

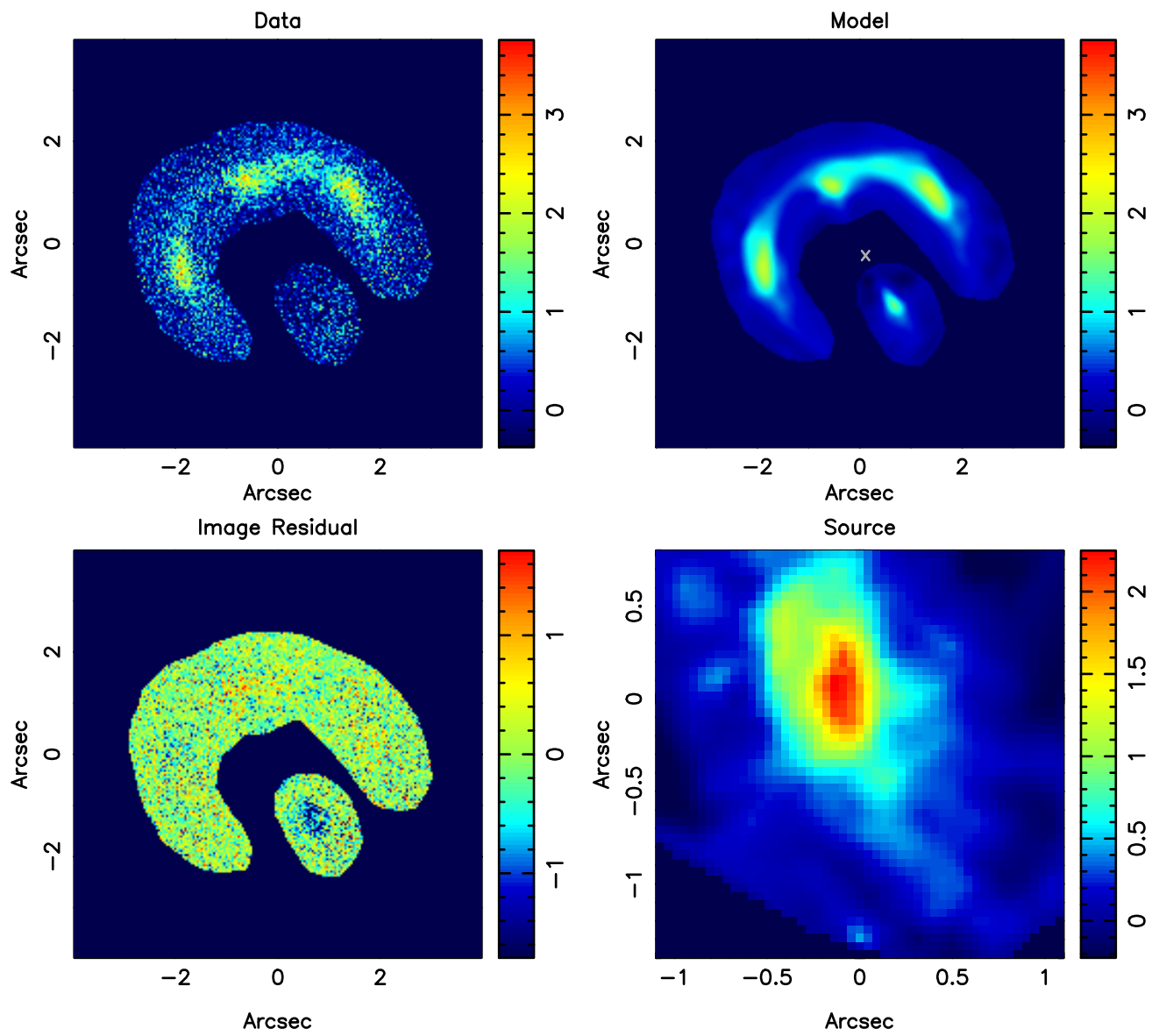

Figure 8. Results from modeling the Keck AO image with a single lens centered on galaxy A, as for Figure 7, but using data after subtraction of both galaxies A and B. (A color version of this figure is available in the online journal.)
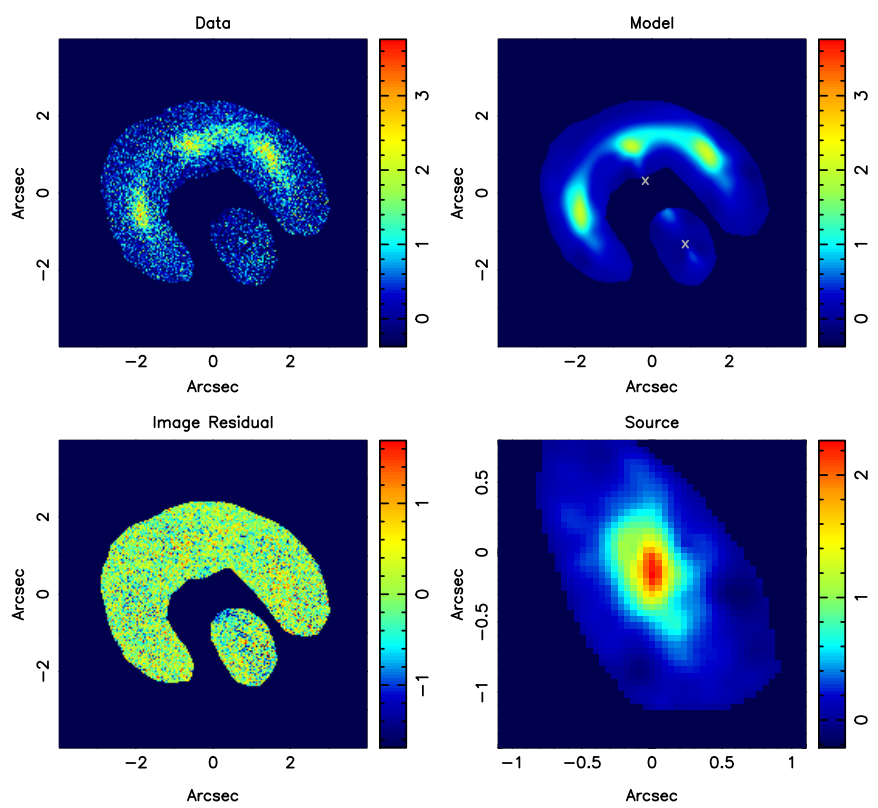

Figure 9. Results from modeling the Keck AO image with a double lens. The regions outside the Einstein half ring and source B are masked and not used for the model fit. North is up and east is to the left. Top left: data after subtraction of A and B. Top right: modeled image. The crosses mark the positions of A and B. There are two very faint objects, one between A and B and one southwest of B; they may be the central image and the counter-image of the arc. Bottom left: residual image, data minus model image. Bottom right: reconstructed image of the source in the source plane.

(A color version of this figure is available in the online journal.)

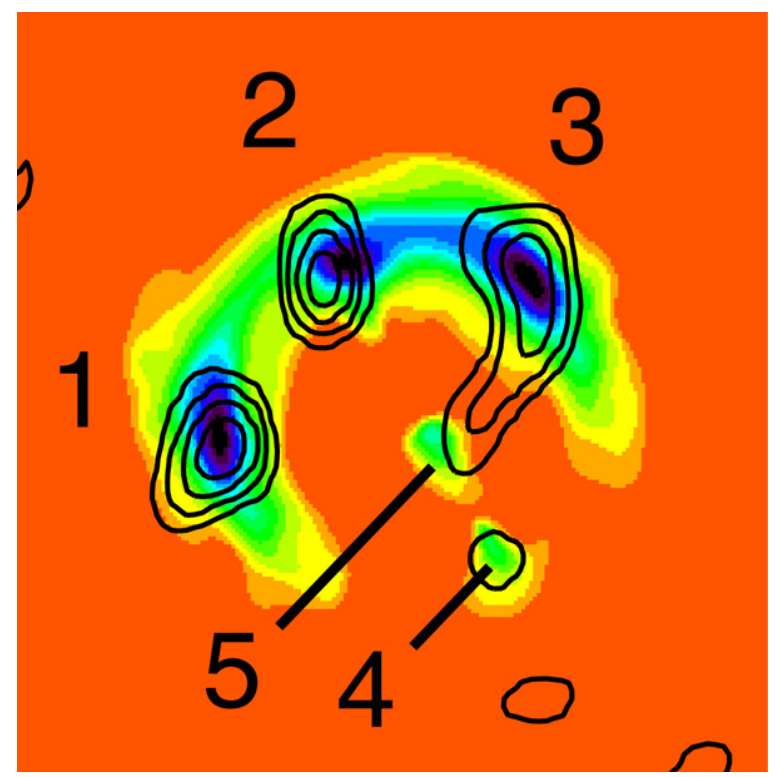

Figure 10. Modeled Keck AO image (color representing surface brightness) based on the double lens. Frame size is 7".5. Superposed contours show the SMA map with the three bright knots labeled. The contours start at $2.5 \sigma$ and increase in steps of $1 \sigma$. Source image 4 coincides with a faint $1 \mathrm{~mm}$ contour. Source image 5 could be indicated by the faint tail of knot 3. Galaxy B lies between knots 4 and 5 .

(A color version of this figure is available in the online journal.) 

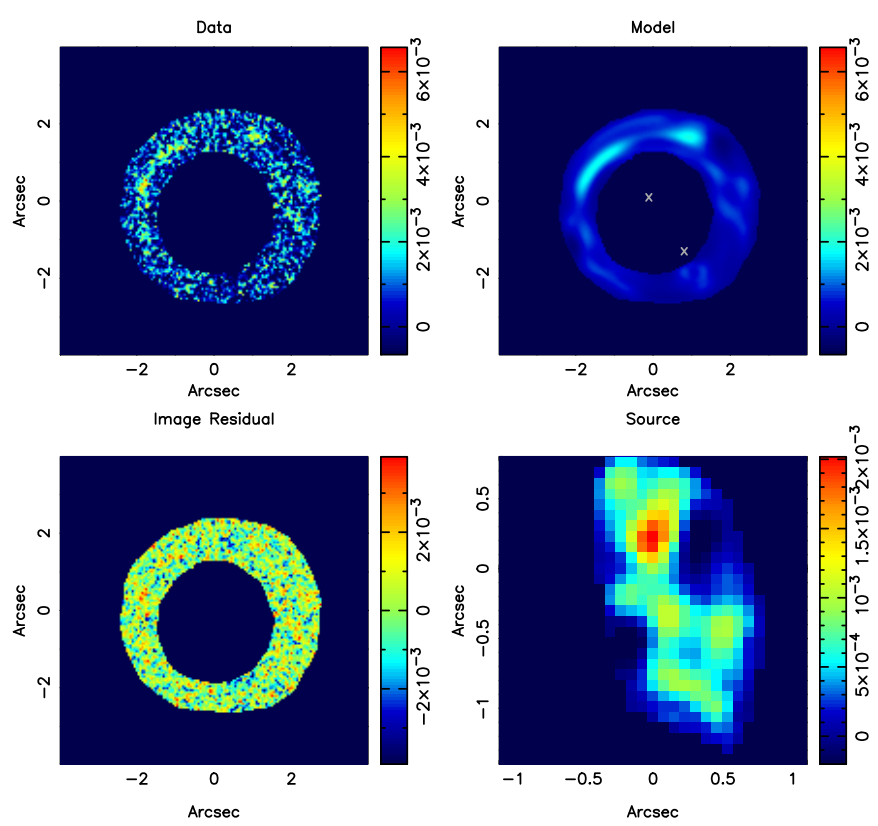

Figure 11. Results from modeling the HST $702 \mathrm{~nm}$ image with the double lens model derived from the Keck AO image. The area other than the Einstein ring is masked out. North is up and east is to the left. Top left: data after subtraction of $\mathrm{A}$ and B. Top right: modeled image. The crosses mark the positions of A and B Bottom left: residual image, data minus model image. Bottom right: reconstructed image of the source in the source plane.

(A color version of this figure is available in the online journal.)

Visual inspection of the residual maps (data - model, Figures 7-9) and the absence of B in the SMA image clearly favor the two-lens model. The statistical quantification (Table 3) agrees. The two-lens model has six extra free parameters allowing us to fit the data better. This is not an artifact of the higher number of free parameters, rather the Bayesian evidence automatically encodes Occam's razor, and models with more free parameters are automatically penalized unless they are really required by the data. In essence, adding six extra parameters to the mass model improves the Bayesian evidence by $\Delta \log E v=300$, which corresponds to about $25 \sigma$ difference, and produces a source galaxy that looks "normal" (Figure 9). If B were not important in the lensing, the model could have set its mass to zero, but this was not the result. ${ }^{21}$

Both the single and double lens models require an external shear of $\sim 0.1$ for lens A (Table 3), suggesting the presence of additional lensing components. While the marginally visible knot 5 (Figure 10) could be the fifth central image, alternatively it

\footnotetext{
21 We have used the Bayesian evidence to determine the best-fit lens model, but for comparison we also give here the more commonly used $\chi^{2}$ values. $\chi^{2}$ values were determined from the residual maps (data minus model) divided by the uncertainty map inside an area of 10912 pixels (Figure 7) However, when source plane pixels are regularized, it is not straightforward to calculate the number of degrees of freedom $N(\mathrm{dof})$. The calculation here excludes the $50 \times$ 50 source pixels in the computation of $N($ dof $)$ ), which is therefore underestimated, thus overestimating the reduced $\chi_{r}^{2}$. With 8 and 14 free parameters for the single- and two-lens models $N($ dof $)=10,904$ and 10,898, respectively. The $\chi^{2}$ values of cases 1,2 , and 3 are 14,393, 12,976, and 12,642, respectively. With these underestimated $N($ dof $)$, the $\chi_{r}^{2}$ values of cases 1,2 , and 3 are $1.32,1.19$, and 1.16 , respectively. In the limit of a large number of degrees of freedom, $N($ dof $) \gtrsim 10,000, \chi_{r}^{2}$ has a standard deviation of $(2 / N(\text { dof }))^{0.5} \approx 0.014$, hence the $\chi_{r}^{2}$ values for case 2 and case 3 differ at the $2 \sigma$ level and are about $10 \sigma$ away from unity. The $\chi_{r}^{2}$ statistic suggests that either a more complex lens model may be needed, or more likely that $N$ (dof) has been underestimated, or that the noise map is underestimated by about $5 \%-10 \%$, which could quite easily be the case.
}
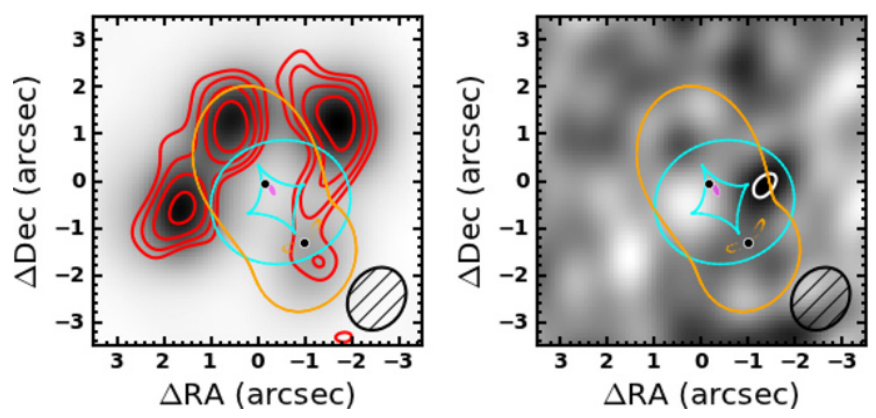

Figure 12. Left: SMA imaging (red contours, starting at $2 \sigma$ and increasing by factors of 1.4) overlaid on the inverted, deconvolved map of the best-fit model visibilities (gray scale). The critical curve and caustics are represented by solid orange and cyan lines, respectively. The position of the lenses and the source are shown by plus signs and a magenta filled ellipse, respectively. The FWHM of the synthesized beam is shown in the lower right corner of each panel. Right: residual image (gray scale) obtained by inverting and deconvolving the residual visibilities (i.e., the difference between the model and data visibilities). Black and white contours indicate $\pm 2 \sigma$ level. A $2 \sigma$ peak in the map of the residual visibilities may be an indication of additional minor structures in the lens plane or in the source plane.

(A color version of this figure is available in the online journal.)

could be a third lensing galaxy $\mathrm{C}$. We checked for this possibility, running a lens model with three lenses centered on $\mathrm{A}, \mathrm{B}$, and C. While $\mathrm{C}$ is still produced by the modeling as an extra image, a bit of mass is removed from $\mathrm{B}$ to $\mathrm{C}$ (Einstein radius of $\mathrm{C}=$ $\left.0 .{ }^{\prime \prime} 030\right)$, and the shear of lens A remains $(\gamma=0.092)$. Also, the Bayesian evidence for the three lens model is poorer than for the two-lens model, and we do not use the three lens model further. Section 5 discusses the implications of the shear.

\subsection{HST Image}

Figure 11 shows the results of applying the double lens model to the HST image. The image residuals are featureless, supporting the adequacy of the model.

\subsection{SMA Image}

For interferometers such as the SMA, proper lens modeling requires the use of visibilities in the Fourier domain rather than surface brightness maps. We followed the technique outlined by Bussmann et al. (2012). Using the Gravlens software ${ }^{22}$, we ray-traced the emission in the source plane to the image plane for a given lensing mass distribution and source morphology. This image plane surface brightness map was then used as input to MIRIAD's UVMODEL task, which computes the Fourier transform of an image and matches the resulting visibilities to the sampling of the observed SMA visibility data set. We used the EMCEE software package (for details, see ForemanMackey et al. 2013) to perform a Markov chain Monte Carlo sampling of the posterior probability density function of our model parameters.

We modeled the SMA data using the lens parameters of the two-lens model derived from the Keck AO data (Table 3) and a Sérsic profile to represent the morphology of the SMG in the source plane. The SMA data (having lower spatial resolution than the KECK AO data) do not justify a more complex model. The best-fit source has a Sérsic index $n_{s}=1.38 \pm 0.46$, halflight radius $r_{s}=1.26 \pm 0.38 \mathrm{kpc}$, axis ratio $b / a=0.52 \pm 0.09$, and P.A. $=20^{\circ} \pm 17^{\circ}$

The best-fit result is shown in Figure 12. Because the model fitting is performed in the Fourier domain and then transformed

\footnotetext{
22 http://redfive.rutgers.edu/ keeton/gravlens/
} 


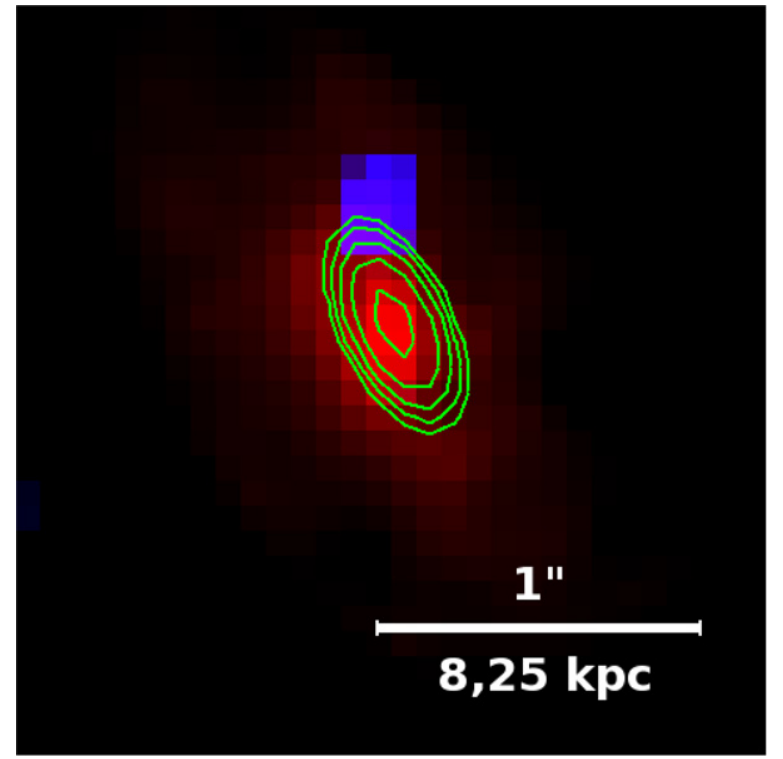

Figure 13. Source image of the SMG reconstructed from the Keck, HST, and SMA images. The stellar emission (Keck $\sim 680 \mathrm{~nm}$ restframe) is shown in red, $\mathrm{UV}(H S T \sim 218 \mathrm{~nm}$ restframe) in blue, and the dust emission (SMA $\sim 310 \mu \mathrm{m}$ restframe) as green contours (logarithmic). North is up and east is to the left.

(A color version of this figure is available in the online journal.)

to the image domain, small residuals in the image domain are not surprising. Modeling the SMA data with and without shear results in equally good fits. The magnification factor at $1 \mathrm{~mm}$ is $\mu=10 \pm 2$.

\section{RECONSTRUCTED SMG MORPHOLOGIES}

The difference in the observed morphologies of the SMG at observed visible, NIR, and millimeter wavelengths (Figure 1) corresponds to morphological differences in the source at the respective restframe wavelengths. Figure 13 shows the multicolor image of the SMG in the source plane as reconstructed from the modeling. Within astrometric errors, the peak of the dust emission (SMA $\sim 310 \mu \mathrm{m}$ restframe) coincides with the stellar light (Keck $\sim 680 \mathrm{~nm}$ restframe). However, compared to the main galaxy body of stars and dust, the bulk of the UV ( $\sim 218 \mathrm{~nm}$ restframe) emission is shifted by $\sim 4 \mathrm{kpc}$ (metric distance at the source) toward the north. Marginal UV emission is also recognizable $\sim 4 \mathrm{kpc}$ south of the galaxy body (Figure 13, and bottom right panel of Figure 11). These extended regions lie closer to the critical curves of the lens model and may be somewhat more magnified than the center of the stellar emission. However, if a strong UV nucleus were present within the compact dust emission, it should be visible in the observed HST image as bright knots similar to those in the SMA image. The HST image shows only the arc.

The offset of the UV emission from the galaxy body of the SMG suggests that the AGN responsible for the high excitation emission lines is located in the galaxy center but hidden from direct view at UV wavelengths. The (apparent) bipolar morphology of the UV emission is reminiscent of the ionization cones seen in the nearby starburst galaxy M82 (Figure 3.108 of Gil de Paz et al. 2007), in Seyfert galaxies (Schmitt et al. 2003), and in high redshift quasars (e.g., CanoDíaz et al. 2012). One interpretation is that the SMG is breaking up its dust cocoon so that UV radiation from the obscured central region escapes through the opened channels and is scattered toward us.
A luminous AGN breaking up its dust cocoon is one interpretation of the so-called alignment effect in PRGs at high redshift (Chambers et al. 1987; McCarthy et al. 1987). In those cases, the breakup is apparently caused by the high-energy particles that create the radio lobes. In contrast to such radio galaxies, the JVLA radio map of 3C 220.3 shows no indication for powerful radio activity in the $\mathrm{SMG}$, either at the known position of lensed features (the arc and the three knots) or from extended lobes, which would be expected to be aligned with the UV emission (approximately north-south). While starburst winds may be able to blow holes into the dust cocoon of the SMG and to expel part of the gas (Tenorio-Tagle \& Munoz-Tunon 1998), a wind from the hidden QSO may be more likely to be forming the open channels. Regardless of the mechanism, the in-progress breakup of the dust cocoon makes the 3C 220.3 SMG an excellent lensed laboratory to explore, with brightness magnification and at higher spatial resolution than possible for unlensed systems, the properties of a dust-enshrouded AGN at high redshift and its feedback on the star forming activity in the host.

\section{THE MASS-TO-LIGHT RATIO OF 3C 220.3}

The double lens model (Table 3) gives total-baryonic and dark-masses for the lensing galaxies $\mathrm{A}$ and $\mathrm{B}$. The calculated masses are $M_{A} \sim 3.5 \times 10^{11} M_{\odot}$ inside a cylinder of Einstein radius $r_{E}=1^{\prime \prime} .02(=7.2 \mathrm{kpc}$ at $z=0.685)$, and $M_{B} \sim 1.2 \times 10^{11} M_{\odot}\left(r_{E}=00^{\prime} \cdot 61=4.2 \mathrm{kpc}\right)$. The statistical uncertainties (Table 3 ), as determined from the model residuals, do not account for possible systematic effects.

One systematic effect for the mass estimates is that the best-fit model requires an external shear of $\sim 0.1$ for lens A (Table 3), suggesting the presence of additional lensing components. This may be consistent with the presence of the $2 \sigma$ residuals of modeling the SMA image (Figure 12). In principle, such a component could be a dark matter halo encompassing A and B. On the other hand, our Chandra image shows no extended X-ray cluster gas (Figure 5), arguing against explaining the shear by a common dark matter halo. More likely is that 3C 220.3 is located in a small group with one or more faint galaxies having escaped detection on our images so far (possibly masked by the lensed structures). A similar situation was reported for the gravitational lens CLASS B2045+265, where the initially inferred high $M / L$ was reduced by a group of faint lensing satellites (Fassnacht et al. 1999; McKean et al. 2007). Therefore, we consider the masses $M_{A}$ and $M_{B}$ as upper limits. ${ }^{23}$

To estimate the luminous mass of galaxies A and B inside the Einstein radius, only two filters (Table 2) provide adequate data. (The spectra show no stellar features.) Using the Starburst99 tool version 6.0.4 (Leitherer et al. 1999) ${ }^{24}$ and adopting a dominant old stellar population typical for radio galaxy hosts (age $\sim$ 3 Gyr and Salpeter initial mass function), we obtain stellar masses $M_{A}^{*} \sim 2.2 \times 10^{11} M_{\odot}$, and $M_{B}^{*} \sim 5.5 \times 10^{10} M_{\odot}$. The uncertainty of these estimates is about a factor of two, dominated by uncertainties in the photometry of A and B inside the Einstein radius (especially from the noisy HST image) and the unknown age of the stellar population. With these numbers, the ratio of dark-to-luminous mass (projected within the Einstein radius) lies in the range between 0.7 and 1.2 , i.e.,

\footnotetext{
$\overline{23}$ The single lens model of 3C 220.3 yields $M_{\text {tot }} \sim 9 \times 10^{11} M_{\odot}$, which is a factor of two higher than the sum of $M_{A}$ and $M_{B}$ in the two-component lens model. If the shear of the two-component lens model is due to further not yet identified lensing galaxies, the mass sum may be reduced further.

24 http://www.stsci.edu/science/starburst99/docs/default.htm
} 


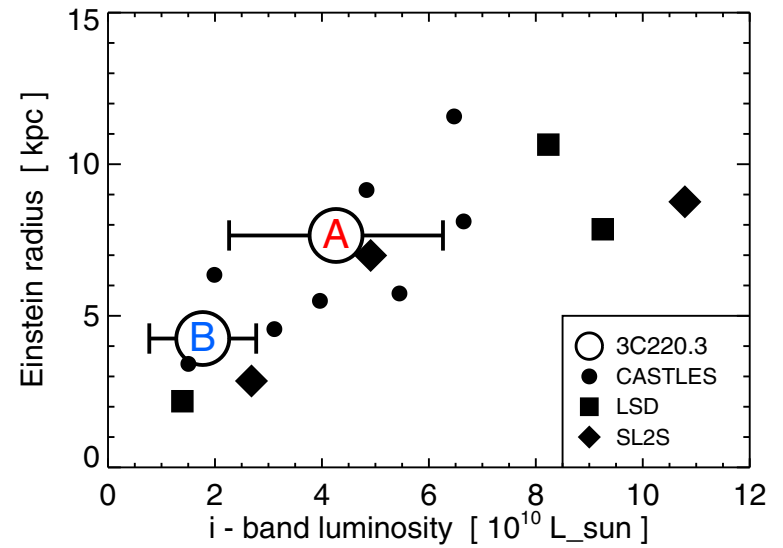

Figure 14. Einstein radius vs. $i$-band luminosity of 3C $220.3 \mathrm{~A}$ and B (large open circles) and the CASTLES, LSD, and SL2S lensing galaxies at comparable redshift (filled symbols).

(A color version of this figure is available in the online journal.)

dark matter fractions $f_{\mathrm{DM}}$ of about $40 \%$ and $55 \%$ with large uncertainties $(\sim 30 \%)$.

To check whether the radio galaxy 3C 220.3 is associated with a usually high amount of dark matter, suitable comparison data are required. A proper comparison sample should contain field galaxies of stellar mass and redshift similar to 3C 220.3. The best existing comparison samples are the CfA-Arizona Space Telescope LEns Survey(CASTLES) ${ }^{25}$ of gravitational lenses (e.g., Lehar et al. 2000) and a few $z \sim 0.6$ objects in the Lenses Structure and Dynamics (LSD) survey (Treu \& Koopmans 2004) and the Strong Lenses in the Legacy Survey (SL2S; Ruff et al. 2011). Figure 14 shows the Einstein radius (converted to kpc) versus the $i$-band luminosity of the CASTLES, LSD, and SL2S lensing galaxies in the redshift range $0.5<z<0.8$, comparable to the redshift of $3 \mathrm{C} 220.3$, and having lensed source redshift $z>1.3$ to ensure that it is sufficiently far from the lens. The $i$-band luminosities of $3 \mathrm{C} 220.3 \mathrm{~A}$ and $\mathrm{B}$ were derived by interpolation of the HST and Keck photometry (Table 2). Assuming the Einstein radius traces total mass, the $M / L$ of 3C $220.3 \mathrm{~A}$ and $\mathrm{B}, M / L(i) \sim 8 \pm 4$, is comparable to that of the CASTLES, LSD, and SL2S lenses, perhaps at the high end of their distribution. LSD and SL2S list dark matter fractions in the range $0.25<f_{\mathrm{DM}}<0.75$ (projected within the Einstein radius), encompassing the value $f_{\mathrm{DM}} \sim 0.4$ of $3 \mathrm{C} 220.3$.

\section{CONCLUSIONS}

The exceptional 3C 220.3 gravitational lensing system consists of an SMG (hosting a dust-enshrouded type-2 QSO) which is lensed by a PRG, i.e., a radio-loud type- 2 quasar. The system permits determination of the total galaxy-scale mass for a powerful double-lobed radio galaxy that is not in a rich galaxy cluster. Comparison with available galaxy-scale lenses indicates an average to moderately high dark matter fraction, but the comparison with radio-quiet galaxies needs to be confirmed with future lens samples near the redshift of 3C 220.3. Further observations of 3C 220.3 with increased $\mathrm{S} / \mathrm{N}$ will allow a more accurate lens model, give a better stellar mass measurement for the lenses, and allow a more detailed reconstruction of the SMG morphology. They should also clarify whether other galaxies important to the lensing, as for example in a small group, exist.

\footnotetext{
25 http://www.cfa.harvard.edu/castles
}

The SMG fits the paradigm of a composite AGN/starburst galaxy in the early universe. The available data indicate the breakup of the dust cocoon, which represents a fundamental transition phase in a violently star-forming galaxy. Deeper X-ray observations and (sub-)millimeter spectroscopy, both possible at high spatial resolution, will allow exploration of the break-up process and the kinematics of any related outflows in a highredshift starburst-AGN.

We thank Shri R. Kulkarni, who enabled us to obtain the Keck LRIS spectrum, and Dan Perley, who assisted with these observations. We also thank Dale Frail for granting Director's Discretionary time at the JVLA, Adam Deller for helping with the JVLA data reduction, and Ray Blundell for granting Director's Discretionary time at the SMA. We thank the referee for comments that improved the paper. Herschel is an ESA space observatory with science instruments provided by European-led Principal Investigator consortia and with important participation from NASA. The Wide-field Infrared Survey Explorer is a joint project of the University of California, Los Angeles and the Jet Propulsion Laboratory/California Institute of Technology. This work is based in part on observations made with the Spitzer Space Telescope, with the Chandra X-ray Observatory, and on observations made with the NASA/ESA Hubble Space Telescope retrieved from the archive at the Space Telescope Science Institute. The National Radio Astronomy Observatory is a facility of the National Science Foundation operated under cooperative agreement by Associated Universities, Inc.

Facilities: Herschel, HST, Spitzer (IRAC, MIPS), EVLA, SMA, Keck:I (LRIS), Keck:II (NIRC2), Hale, CXO

\section{REFERENCES}

Auger, M. W., Treu, T., Bolton, A. S., et al. 2009, ApJ, 705, 1199

Belsole, E., Worrall, D. M., Hardcastle, M. J., \& Croston, J. H. 2007, MNRAS, 381, 1109

Bussmann, R. S., Gurwell, M. A., Fu, H., et al. 2012, ApJ, 756, A134

Cano-Díaz, M., Maiolino, R., Marconi, A., et al. 2012, A\&A, 537, L8

Chambers, K. C., Miley, G. K., \& van Breugel, W. 1987, Natur, 329, 604

Chapman, S. C., Blain, A. W., Smail, I., \& Ivison, R. J. 2005, ApJ, 662, 772

Cleary, K., Lawrence, C. R., Marshall, J. A., Hao, L., \& Meier, D. 2007, ApJ, 660,117

Comerford, J. M., Meneghetti, M., Bartelmann, M., \& Schirmer, M. 2006, ApJ, 642,39

Dickinson, M. 1993, in ASP Conf. Ser. 51, Observational Cosmology, ed. G. L. Chincarini, A. Iovino, T. Maccacaro, \& D. Maccagni (San Francisco, CA: ASP), 434

Dickinson, M. 1994, PhD thesis, Univ. California Berkeley

Fassnacht, C. D., Blandford, R. D., Cohen, J. G., et al. 1999, AJ, 117, 658

Fazio, G., Hora, J. L., Allen, L. E., et al. 2004, ApJS, 154, 10

Foreman-Mackey, D., Hogg, D. W., Lang, D., \& Goodman, J. 2013, PASP, 125,306

Gil de Paz, A., Boissier, S., Madore, B. F., et al. 2007, ApJS, 173, 185

Griffin, M. J., Abergel, A., Abreu, A., et al. 2010, A\&A, 518, L3

Keeton, C. R. 2001, ApJ, 561, 46

Koopmans, L. V. E. 2005, MNRAS, 363, 1136

Lehar, J., Falco, E. E., Kochanek, C. S., et al. 2000, ApJ, 536, 584

Leitherer, C., Schaerer, D., Goldader, J. D., et al. 1999, ApJS, 123, 3

Lilly, S. J., \& Longair, M. S. 1984, MNRAS, 211, 833

Magnelli, B., Lutz, D., Santini, P., et al. 2012, A\&A, 539, 155

Massey, P., \& Gronwall, C. 1990, ApJ, 358, 344

McCarthy, P. J. 1993, ARA\&A, 31, 639

McCarthy, P. J., Miley, G. K., de Koff, S., et al. 1997, ApJS, 112, 415

McCarthy, P. J., van Breugel, W., Spinrad, H., \& Djorgovski, S. 1987, ApJL, 321, L29

McKean, J. P., Koopmans, L. V. E., Flack, C. E., et al. 2007, MNRAS, 378, 109

Mullin, L. M., Hardcastle, M. J., \& Riley, J. M. 2006, MNRAS, 372, 113

Negrello, M., Hopwood, R., De Zotti, G., et al. 2010, Sci, 330, 800

Oke, J. B., Cohen, J. G., Carr, M., et al. 1995, PASP, 107, 375

Ota, N., Mitsuda, K., Hattori, M., \& Mihara, T. 2000, ApJ, 530, 172 
Ott, S. 2010, in ASP Conf. Ser. 434 Astronomical Data Analysis Software and Systems XIX, ed. Y. Mizumoto, K.-I. Morita, \& M. Ohishi (San Francisco, CA: ASP), 139

Pilbratt, G. L., Riedinger, J. R., Passvogel, T., et al. 2010, A\&A, 518, L1

Poglitsch, A., Waelkens, C., Geis, N., et al. 2010, A\&A, 518, L2

Pratt, G. W., Croston, J. H., Arnaud, M., \& Böhringer, H. 2009, A\&A, 498, 361

Rieke, G. H., Young, E. T., Engelbracht, C. W., et al. 2004, ApJS, 154, 25

Ruff, A. J., Gavazzi, R., Marshall, P. J., et al. 2011, ApJ, 727, 96

Sault, R. J., Teuben, P. J., \& Wright, M. C. H. 1995, ASPC, 77, 433

Schmitt, H. R., Donley, J. L., Antonucci, R. R. J., Hutchings, J. B., \& Kinney, A. L. 2003, ApJS, 148, 327

Schuster, M. T., Marengo, M., \& Patten, B. M. 2006, Proc. SPIE, 6270, 65
Smail, I., \& Dickinson, M. 1995, ApJL, 455, L99

Spinrad, H., Marr, J., Aguilar, L., \& Djorgovski, S. 1985, PASP, 97, 932

Stern, D., Dey, A., Spinrad, H., et al. 1999, AJ, 117, 1122

Stern, D., Moran, E. C., Coil, A. L., et al. 2002, ApJ, 568, 71

Suyu, S. H., Marshall, P. J., Hobson, M. P., \& Blandford, R. D. 2006, MNRAS, 371,983

Tenorio-Tagle, G., \& Munoz-Tunon, C. 1998, MNRAS, 293, 299

Treu, T., \& Koopmans, L. V. E. 2004, ApJ, 611, 739

Vegetti, S., \& Koopmans, L. V. E. 2009, MNRAS, 392, 945

Werner, M. W., Roellig, T. L., Low, F. J., et al. 2004, ApJS, 154, 1

White, S. D. M., \& Rees, M. J. 1978, MNRAS, 183, 341

Wright, E. L., Eisenhardt, P. R. M., Mainzer, A. K., et al. 2010, AJ, 140, 1868 